\title{
Description and Performance of the Preamplifier for the National Ignition Facility (NIF) Laser System
}

\author{
J. K. Crane, M. Martinez, B. Moran, \\ C. Laumann, J. Davin, R. Beach, \\ B. Golick, R. Jones, J. Braucht, \\ M. Perry, K. Skulina, F. Penko,
}

S. Herman, S. Burkhart, and S. Mitchell

This paper was prepared for submittal to the 2nd Annual International Conference on Solid-State Lasers for Application to Inertial Confinement Fusion Paris, France October 22-25, 1996

December 19, 1996

This is a preprint of a paper intended for publication in a journal or proceedings. Since changes may be made before publication, this preprint is made available with the understanding that it will not be cited or reproduced without the permission of the author. 


\section{DISCLAIMER}

This document was prepared as an account of work sponsored by an agency of the United States Government. Neither the United States Government nor the University of California nor any of their employees, makes any warranty, express or implied, or assumes any legal liability or responsibility for the accuracy, completeness, or usefulness of any information, apparatus, product, or process

disclosed, or represents that its use would not infringe privately owned rights. Reference herein to any specific commercial product, process, or service by trade name, trademark, manufacturer, or otherwise, does not necessarily constitute or imply its endorsement, recommendation, or favoring by the United States Government or the University of California. The views and opinions of authors expressed herein do not necessarily state or reflect those of the United States Government or the University of California, and shall not be used for advertising or product endorsement purposes. 
Description and performance of the preamplifier for the National Ignition Facility (NIF) laser system

John K. Crane, Mikael Martinez, Bryan Moran, Curt Laumann, James Davin, Raymond Beach, Brad Golick, Russ Jones, John Braucht, Michael Perry, Kenneth Skulina, Frank Penko, Steve Herman, Scott Burkhart, and Scott Mitchell

L-490, Lawrence Livermore National Laboratory, P. O. Box 808, Livermore, CA 94550

\section{ABSTRACT}

We describe the prototype preamplifier for the NIF laser system and discuss the performance of the regenerative amplifier and 4-pass laser systems that comprise the preamplifier.

\section{INTRODUCTION}

The preamplifier for the NIF laser system takes the $1 \mathrm{~nJ}$, shaped and modulated pulses generated in the master oscillator room, amplifies them to the $12 \mathrm{~J}$ level, spatially shapes them to precompensate for the gain profile of the main amplifiers and adds an angular dither to the beam at the phase modulation frequency to remove spatial coherence. Four subsystems form the preamplifier: a diode-pumped, Nd:glass regenerative amplifier; a spatial shaping optical system, an optical system for smoothing by spectral dispersion ( SSD ), and a $45 \mathrm{~mm}$ rod amplifier. All four of these optical subsystems, shown in Figure 1, will be housed in the preamplifier module (or PAM) along with the energizing, control, and diagnostic instrumentation.

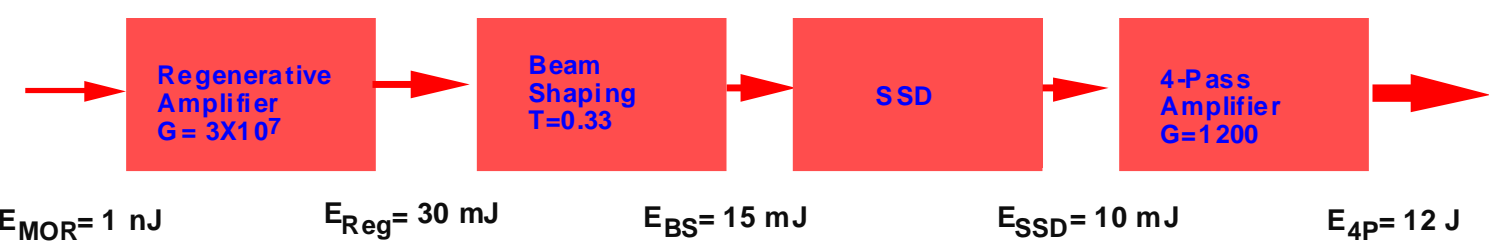

Figure 1. Block diagram of the PAM, showing the component subsystems and the energy at each subsystem interface.

The current specifications ${ }^{1}$ for the laser pulse at the injection plane of the main amplifiers are shown in Table I.

$\begin{array}{ll}\text { Energy } & 3 \mathrm{~J} \\ \text { Power } & 1.2 \mathrm{GW} \\ \text { Temporal contrast } & 100: 1 \\ \text { Spatial profile } & 2: 1 \\ \text { Phase front } & \text { Less than } 0.15 \% \text { of the total beam power is } \\ & \text { in the spatial frequency band: } \\ & 0.7 \mathrm{~mm}^{-1} \leq \mathrm{v} \leq 4 \mathrm{~mm}^{-1} \\ \text { RMS power stablilty } & 3 \% \\ \text { Pulse duration } & 21 \mathrm{~ns} \\ \text { Prepulse } 20 \text { ns prior to main pulse (relative to main } & 10^{-5} \text { to } 10^{-6} \\ \text { pulse energy/power) } & \\ \text { Near field spatial contrast } & \leq 5 \%\end{array}$

Table 1. Requirements for the NIF laser system at the injection plane of the transport spatial filters.

The output performance specifications of the PAMs must satisfy these requirements plus provide additional margin in energy and power to account for transport loss from the PAM output to the injection plane of the transport spatial filters, and to allow for shortfalls in main amplifier performance over the life of the laser system. In the following sections we will describe the design and performance of the regenerative amplifier and 4-pass power amplifier that are currently being prototyped for the NIF PAMs. 


\section{REGENERATIVE AMPLIFIER}

The regenerative amplifier is a folded-linear, $\mathrm{TEM}_{00}$ cavity that must be able to contain a $21 \mathrm{~ns}$ long, temporally-shaped pulse. At either end of the cavity are diode-pumped, $5 \times 50 \mathrm{~mm}$ rod amplifiers that are end pumped by $48 \mathrm{bar}$, diode arrays. The diode light is focused with micro-lenses into a tapered lens duct that funnels light down into the end of the $5 \mathrm{~mm}$ diameter laser rod. ${ }^{2}$ Each rod stores about $300 \mathrm{~mJ}$ of energy in the upper laser state from $3 \mathrm{~kW}$ of optical pump power in a $350 \mu$ s pulse. The total available energy in the cavity eigenmode is about $60 \mathrm{~mJ}$. The $1 \mathrm{~nJ}$ input pulse is amplified to $10 \mathrm{~mJ}$ in about 15 round trips in the regen, requiring a total gain of $10^{7}$. To preserve the specific temporal shape of the input pulse, the regen is limited to a square-pulse-distortion of 1.2. To achieve an acceptable energy balance among the 192 arms of the laser, the regen must have a pulse-to-pulse stability < 3\% rms.

Figure 2 shows the current design of the NIF regenerative amplifier, including the optics for seeding the regen with a pulse from the master oscillator room (MOR). Light from the MOR propagates over single-mode, polarization-preserving fiber and is introduced into the preamplifier with a fiber launch. Two Faraday isolators provide $60 \mathrm{~dB}$ of isolation between the fiber output and the regen in order to protect the fiber from the Q-switched output of the regen and to reduce feedback into the MOR from the preamplifier. The launch optics include a two lens telescope that matches the output mode of the fiber to the cavity eigenmode of the regen. The seed pulse reflects from the input thin-film polarizer (TFP) into the cavity of the regenerative amplifier.

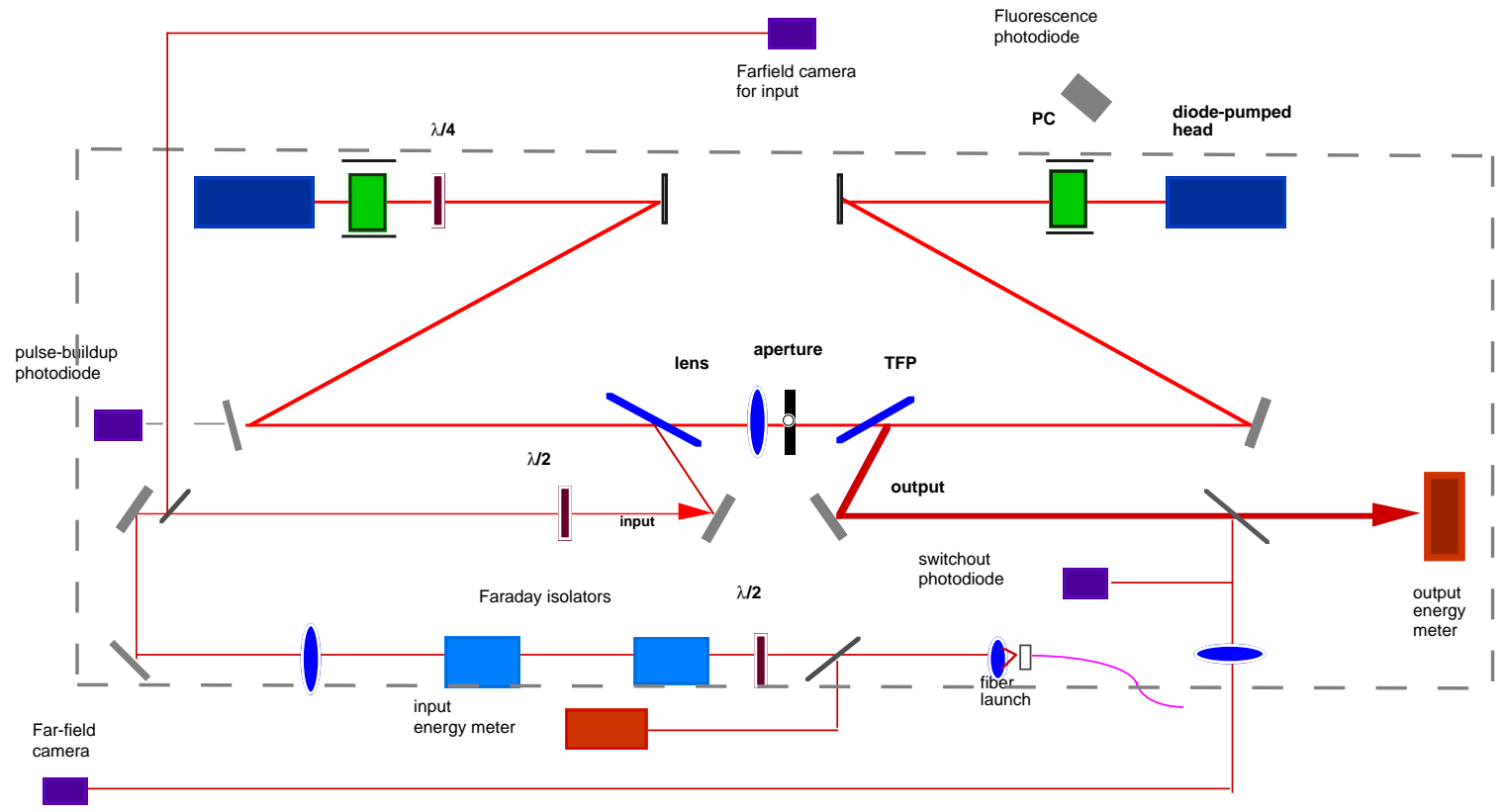

Figure 2. Layout of the regenerative amplifier prototype, including regen cavity, launch optics, and various diagnostics.

Once in the cavity, the s-polarized input pulse makes an initial round trip with both Pockels cells turned off. When the entire length of the pulse has double-passed the quarter-wave plate and Pockels cell on the left-hand side of the cavity, the pulse is converted to p-polarization and the Pockels cell turns on to add an additional quarter-wave of phase retardation. The combination of the static quarter wave from the waveplate and the quarter wave from the energized Pockels cell effectively cancel and the circulating pulse remains ppolarized until switchout. When the pulse has reached the desired energy level the second Pockels cell on the right-hand side of the cavity is energized and the pulse is converted to s-polarization and reflects out of the cavity from the output thin-film polarizer. The layout shows a variety of diagnostics that are used to look at output energy, far-field beam profile, pulse buildup in the cavity, output pulse shape, and amplifier fluorescence.

The cavity is designed to be a stable, $\mathrm{TEM}_{00}$, nearly confocal cavity that has a round trip transit time of $30 \mathrm{~ns}$. This cavity length allows the $21 \mathrm{~ns}$ pulse to be entirely contained in the cavity with 
additional time for clean switchin and switchout of the shaped pulse. The $450 \mathrm{~cm}$ long cavity has a $200 \mathrm{~cm}$ focal length lens located at the midway point between the ends; the value of the stability parameter, $g=0.1$, places the cavity near the center of the stability diagram. ${ }^{3}$ We place an iris next to the lens that we can adjust to suppress higher order spatial modes. To diagnose single spatial mode operation we run the regen in a long pulse mode and observe the relaxation oscillations using a photodiode. When the cavity allows lasing on higher spatial modes the relaxation oscillations show multiple frequency components; single spatial mode oscillation exhibits single frequency relaxations. Examples of single and multiple spatial mode behavior are shown in Fig. 3.

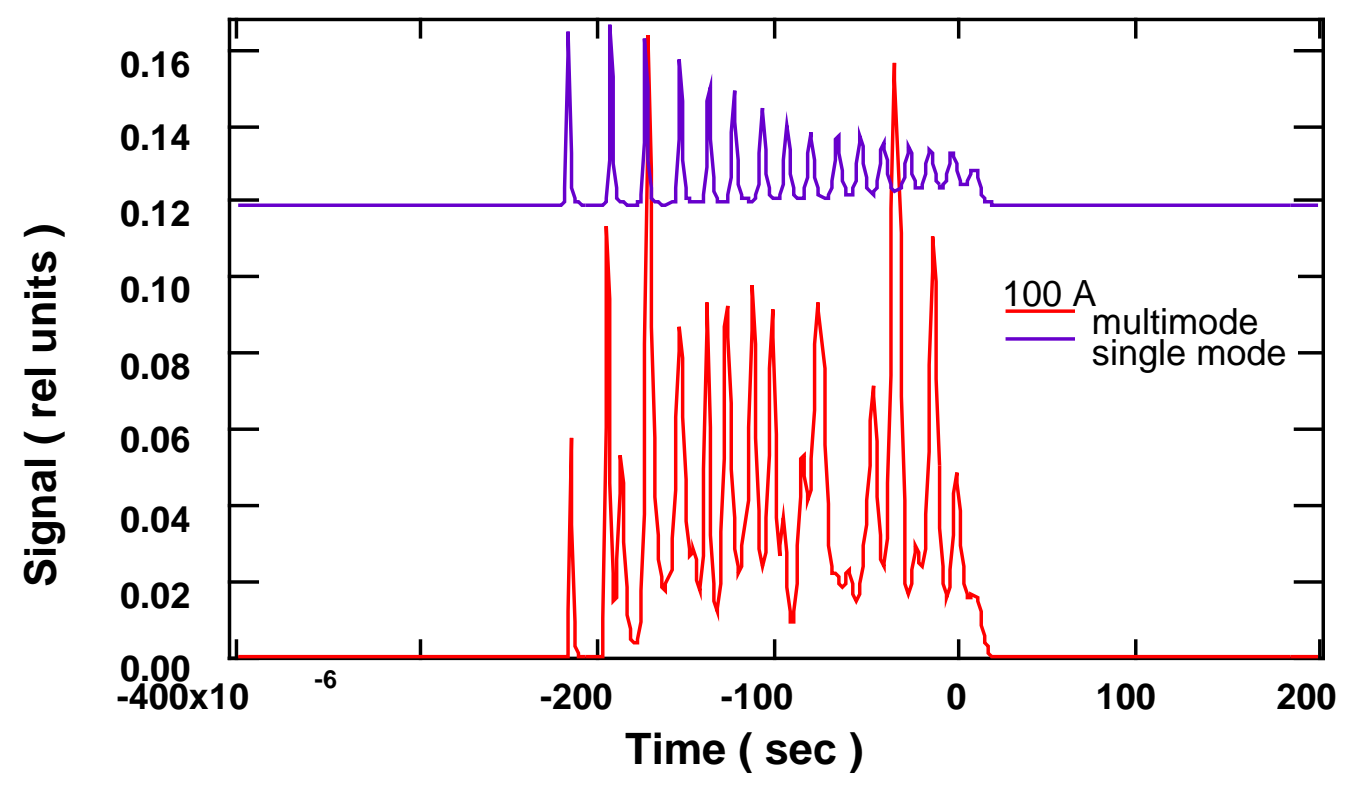

Figure 3. Relaxation oscillations observed when operating the regen cavity as a long pulse laser.

When the quarter-wave plate near the left-hand side of the cavity is adjusted to add $\pi / 2$ retardation between orthogonal polarization components, long pulse operation is suppressed and the cavity can be Qswitched by energizing the Pockels cell. When unseeded, laser power will buildup from spontaneous emission noise in the Q-switched cavity at a rate determined by the net round trip gain. If a seed pulse is switched into the cavity at the appropriate time the pulse buildup can be much faster, depending on the ratio of the seed power to the unseeded noise power. The temporal shift between the seeded and unseeded cavity buildup can be used as a measure of the signal-to-noise ratio at the regen input. Figure 4 shows a photodiode signal looking into the cavity at pulse buildup for seeded and unseeded operation. We can write expressions for the circulating power, $\mathrm{P}$, in the cavity as a function of cavity round trip, $\mathrm{k}$, gain, $\mathrm{G}$, and cavity transmission, $\mathrm{T}$ :

$$
\mathrm{P}=\mathrm{S} \bullet(\mathrm{G} \bullet \mathrm{T})^{\mathrm{k}} .
$$

If we measure the temporal shift between seeded and unseeded operation at equal power points on the cavity power signal (Fig. 4) then we can derive the signal-to-noise ratio, S/N.

$$
\begin{aligned}
\mathrm{P}_{\text {seed }}(\mathrm{k})=\mathrm{S} \bullet(\mathrm{G} \bullet \mathrm{T})^{\mathrm{k}} & =\mathrm{N} \bullet(\mathrm{G} \bullet \mathrm{T})^{\mathrm{m}}=\mathrm{P}_{\text {noise }}(\mathrm{m}) \\
\mathrm{S} / \mathrm{N} & =(\mathrm{G} \bullet \mathrm{T})^{\mathrm{m}-\mathrm{k}}
\end{aligned}
$$

In the example shown in Fig. $4, \mathrm{~S} / \mathrm{N}=2.7 \mathrm{X} 10^{4}$. 


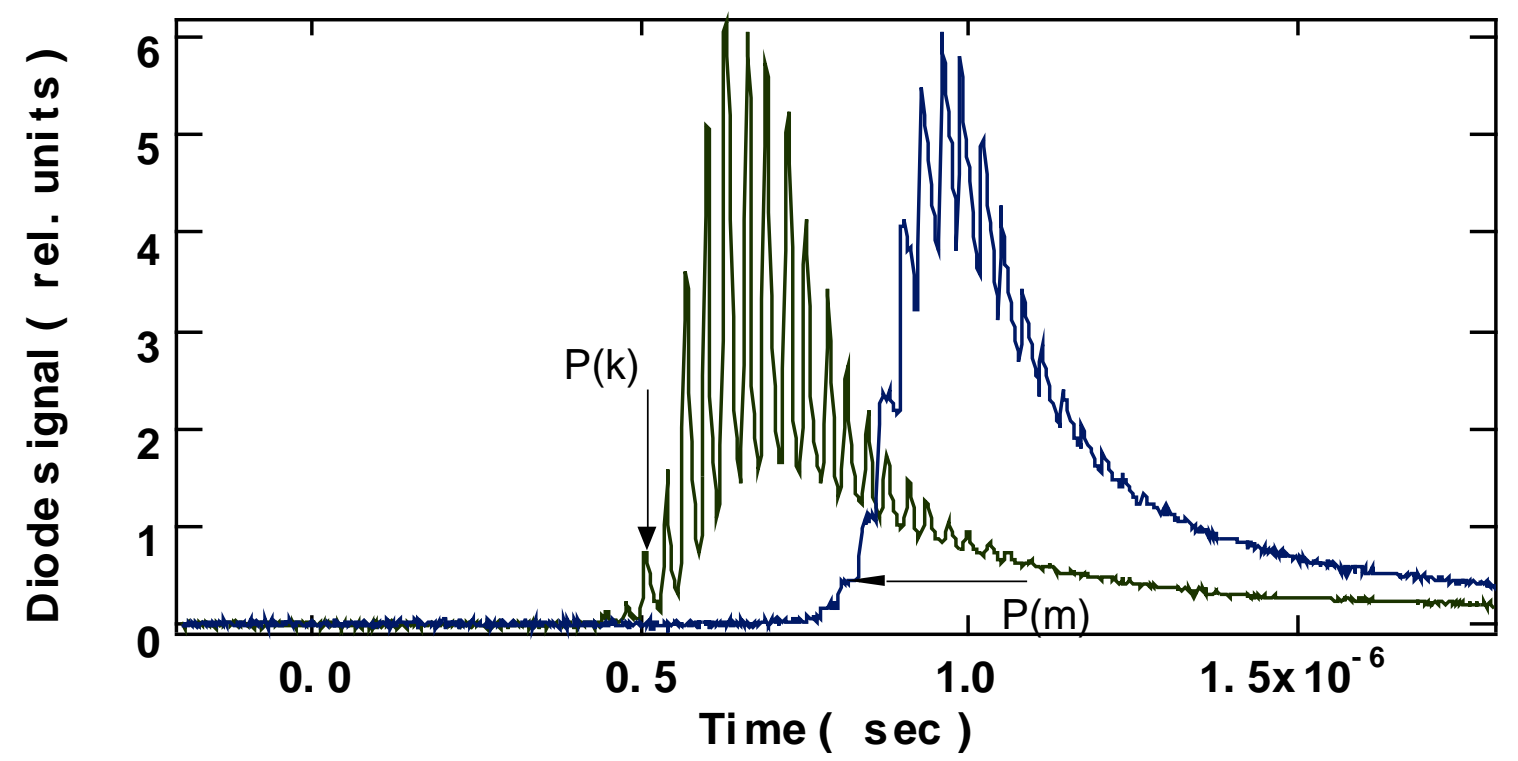

Figure 4. Photodiode signal showing laser power buildup in the cavity for unseeded (blue) and seeded (green), Q-switched operation.

We have developed an energetics model for the regenerative amplifier based upon the classical coupled cavity-atom description of the laser. ${ }^{4}$ This model allows us to optimize the current regenerative amplifier design, make performance predictions for cavity or gain medium modifications, and to analyze experimental results for the regen and 4-pass amplifiers. The model is based upon the time-dependent equation for gain 5 , G(t),

$$
\mathrm{G}(\mathrm{t})=1 /(1-(1-(1 / \mathrm{G}(0)) \bullet \exp (-\mathrm{U}(\mathrm{t}) / \mathrm{Jsat}))
$$

where Jsat is the saturation fluence of the laser glass, $G(0)$ is the initial gain, and

$$
U(t)=\int_{t} I\left(t^{\prime}\right) d t^{\prime}
$$

In equation $(5) \mathrm{I}(\mathrm{t})$ is the pulse irradiance and $\mathrm{U}(\mathrm{t})$ is a running value for fluence at time " $\mathrm{t}$ ". As a test we compared the model results with the experimentally measured power buildup in the cavity and the pulse switched out of the cavity. Both power measurements were made using fast photodiodes: the cavity buildup photodiode was positioned to measure leakage behind the final cavity mirror before the output thin film polarizer, and the second photodiode looked at the pulse after switchout from the cavity. Both photodiodes were calibrated against an energy meter at the output. Figure 5a. shows the diode signals in units of power and the model prediction for regen power at the values of gain and cavity loss corresponding to the operating point of the amplifiers. There is good agreement between the model and data for this time-dependent calculation. Figure 5b shows measurements of the temporal pulse distortion for different values of amplifier gain, comparing model to measurements. For our current cavity configuration we can expect to extract about $10 \mathrm{~mJ}$ and still maintain a pulse shape distortion (PSD) of 1.2. To extract significantly more energy at the same value of PSD we must reduce the round trip loss in the cavity, $\mathrm{L}=0.30$ per round trip, or enlarge the spatial mode in the rod, $\mathrm{w}_{0}=0.10 \mathrm{~mm}$ ( $1 / \mathrm{e}^{2}$ radius $)$. 


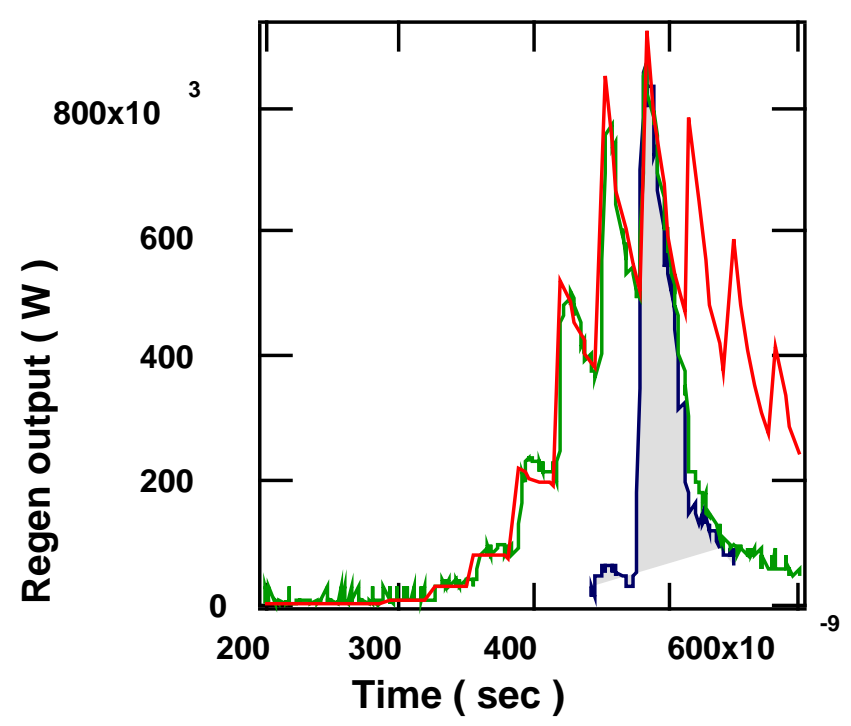

(a.)

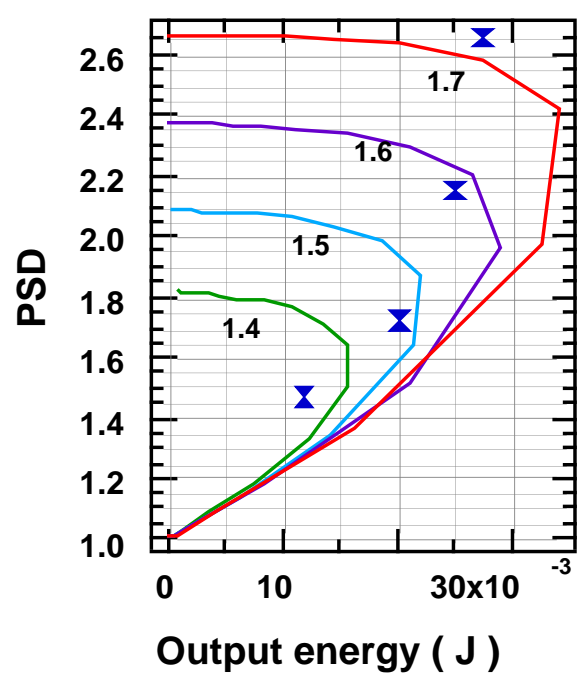

(b.)

Figure 5. (a).Comparison between measured buildup of cavity power (solid curve) and the model prediction (dashed curve). The dotted, filled curve is a switched out pulse of $22 \mathrm{~mJ}$. (b). Model results showing squarepulse-distortion versus output energy for different values of single pass gain: 1.4,1.5,1.6, and 1.7 in each amplifier; hour glass symbols represent measured values.

\section{MULTIPASS AMPLIFIER}

The laser pulse exiting the regen is $10-30 \mathrm{~mJ}$ in energy in a Gaussian-shaped spatial mode. Immediately after exiting the regen the pulse propagates through another Pockels cell where any pulses leading or trailing the main pulse are sliced off. Next the $3 \mathrm{~mm}$ diameter pulse is magnified in a three element adjustable telescope that has a magnification range of 10-30X. This expanded beam overfills a square, apodizing filter with serrations that define the fill factor of the resulting square, super-Gaussian beam that is amplified in the four-pass amplifier. The layout of the four-pass amplifier is shown in Figure 6.

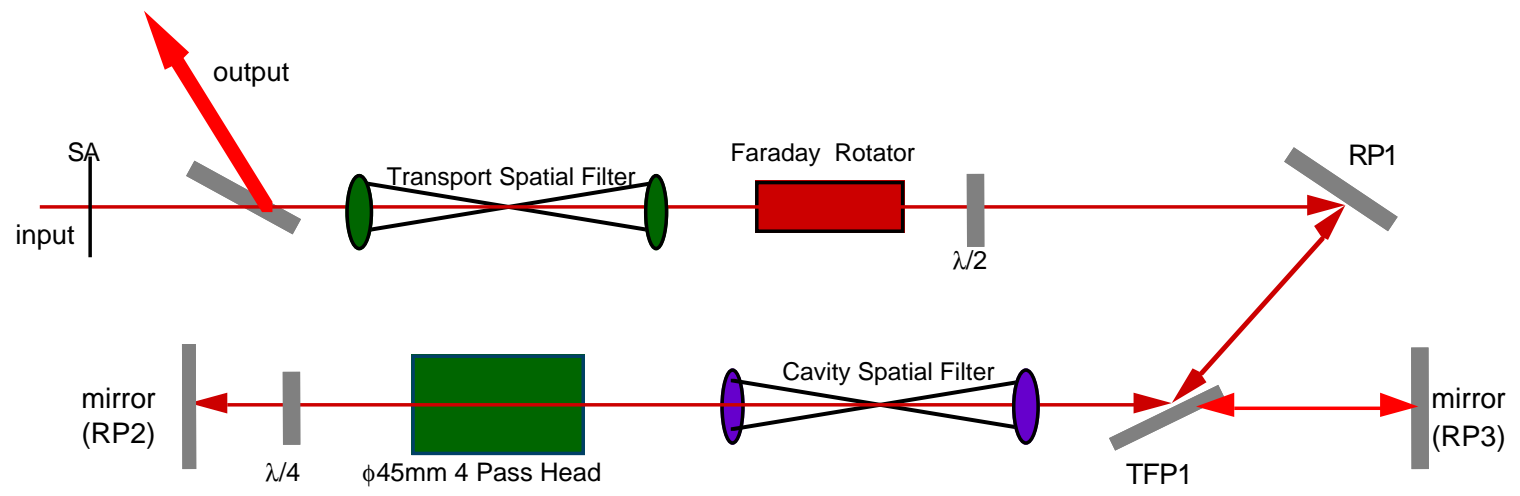

Figure 6. Optical layout of the 4-pass amplifier

The input pulse is p-polarized and transmits through the thin-film polarizer. A transport spatial filter is the first telescope in the afocal image relay optical system. This telescope has two pinholes that are $2 \mathrm{~mm}$ in diameter and spaced by $5 \mathrm{~mm}$. The first pinhole encountered by the input pulse strips away the higher order spatial components imposed on the beam by the serrations of the input apodizer. The next two elements are a Faraday rotator and half-wave plate which rotate the polarization of the light from "p" to "s". A mirror, located at the first relay plane deflects the light to a thin-film polarizer, TFP1, that reflects the pulse into 
the four-pass cavity. The cavity contains the flashlamp pumped, $45 \mathrm{~mm}$ rod amplifier and a second relay telescope with four pinholes arranged in a square pattern, $5 \mathrm{~mm}$ on a side. The incoming s-polarized pulse passes through the first pinhole, passes through the amplifier, then the quarter-wave plate and is reflected back by the left-hand cavity mirror located at the second relay plane. In the second pass the light propagates back through the cavity at a slightly different angle and so passes through a different pinhole in the cavity spatial filter. The polarization has been converted from "s" to " $p$ " by the quarter-wave plate and so passes through TFP1 and is reflected back by the right-hand cavity mirror at relay plane 3, rp3. The third and fourth passes of the cavity go through the remaining pinholes. After the fourth pass through the telescope the s-polarized light reflects from TFP1 back out of the cavity. The combination of half-wave plate and Faraday rotator now cancel and the light remains s-polarized and is reflected off the input thin-film polarizer as shown in Fig. 6.

\section{$3.145 \mathrm{~mm}$ amplifier}

The flashlamp pumped rod amplifier was developed at Lawrence Livermore National Laboratory for the Petawatt and NIF Laser systems. The 45X220 mm cylindrical rod is lightly Nd-doped (0.4-0.55\%) LHG-8 glass inside a diffuse, cylindrical reflector made of the ceramic material Accuratus. Twelve Ce-doped, xenon-filled flashlamps are positioned around the rod to provide a radial-dependent gain profile. The twelve flashlamps are driven by two, parallel circuits that deliver $25 \mathrm{~kJ}$ of electrical energy at $16 \mathrm{kV}$ charge voltage in a 350 microsecond pulse. The lamps are preionized with a lower energy pulse that is fired about 200 microseconds before the main pulse. Figure 7 shows data on the gain profile and electrical waveforms for the $45 \mathrm{~mm}$ rod amplifier. We measured the gain using a small probe beam centered at the peak of the spectral gain curve for LHG8 glass.

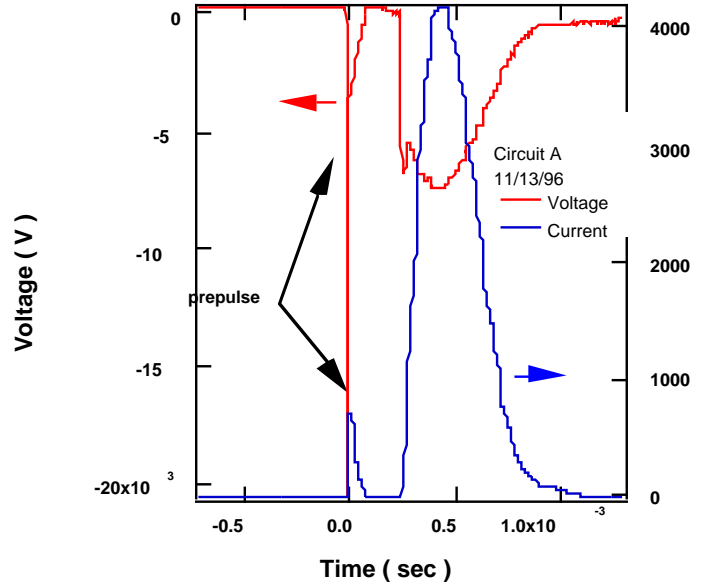

(a.)

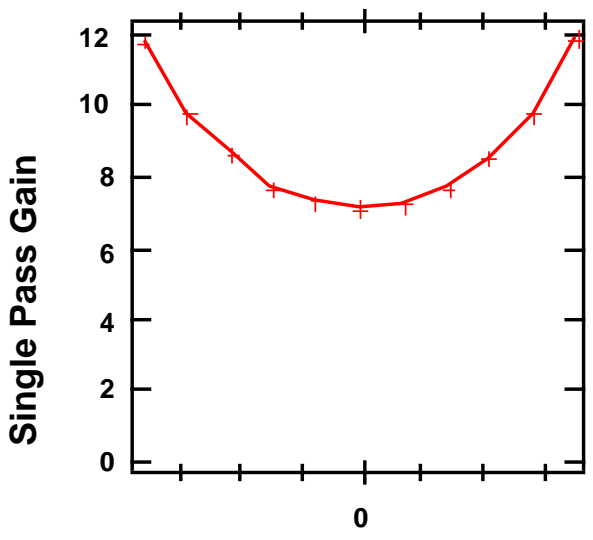

Radial distance ( $\mathrm{mm}$ )

(b.)

Figure 7. (a). Voltage and current waveforms measured at amplifier electrodes. (b). Radial gain profile for $0.55 \%$ doped rod with $25 \mathrm{~kJ}$ input.

\subsection{Performance of 4-pass amplifier}

We measured the output energy of the 4-pass amplifier as a function of input energy from the regen and input electrical energy to the $45 \mathrm{~mm}$ amplifier. The input energy was measured directly after the serrated aperture using a photodiode calibrated to a calorimeter. To measure the output energy we used a large aperture Scientech calorimeter. We set up cameras to look at the near-field beam images at the input and output of the 4-pass and fast photodiodes to measure the temporal shape at the 4-pass input and output. Before performing these measurements we added various light baffles to suppress self-oscillation in the 4pass cavity, which resulted from feedback from pinhole plates and optical mounts. Figure 8 shows results of the measurements for output energy vs input energy at different charge voltages. Along with the data we plot the results of a model fit based upon the Frantz-Nodvik equation given by Eq.(4). We have obtained up to $14 \mathrm{~J}$ out of the 4-pass amplifier for an input energy of $15 \mathrm{~mJ}$ at a charge voltage of $14 \mathrm{kV}$. 


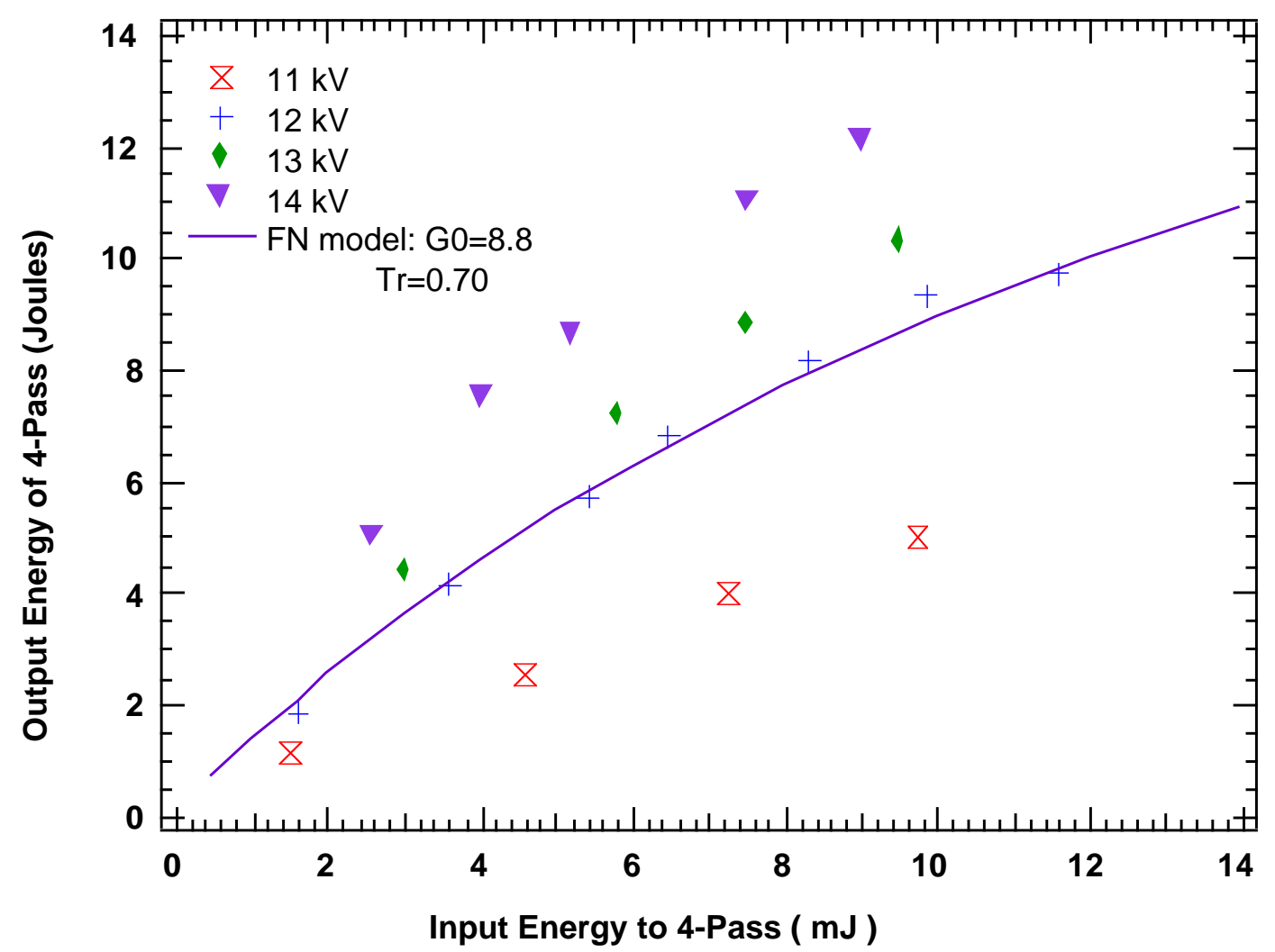

Figure 8. Four-pass output energy vs input energy for different charge voltages. The solid line gives a model fit using a single-pass gain of 8.8 and a transmission of 0.70 .

We have attempted to produce a spatially flat output beam by varying the size of the magnified Gaussian beam at the serrated aperture to compensate for the rods radial gain profile. If successful this technique can reduce the energy loss incurred from shaping the beam with neutral density masks. The image in Fig. 9 represents an attempt to optimize flatness at the output of the four-pass by adjusting the magnification of the telescope that magnifies the beam in the shaping section between regen and four-pass. To produce a symmetric intensity distribution we took special care to accurately align the amplifier rod to the incoming beam using cross-hairs. The histogram shows the distribution of intensities and the ratio, $\Delta \mathrm{I} /\langle\mathrm{I}\rangle=0.14$ for this data set (standard deviation over the mean). The results for different size Gaussian beams illuminating the serrated aperture were similar. The output beam always reveals corners that vary in intensity compared to the central portion of the beam. This limits our ability to shape the beam to produce a uniformly square pattern without additional masks. This effect is expected and is a result of amplifying a square beam in a cylindrical rod that has a radially dependent gain profile (see Fig. 7b). 


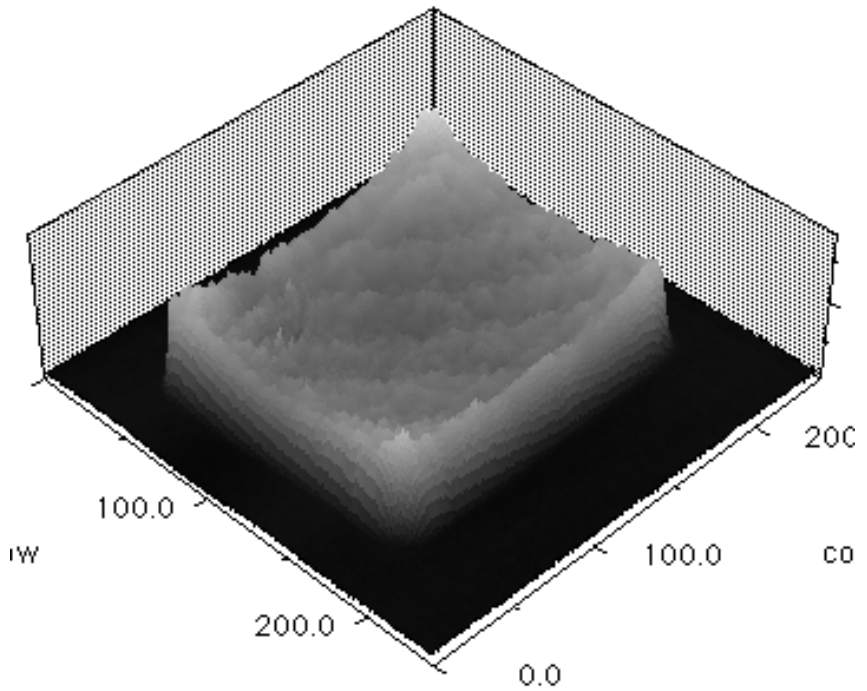

a.

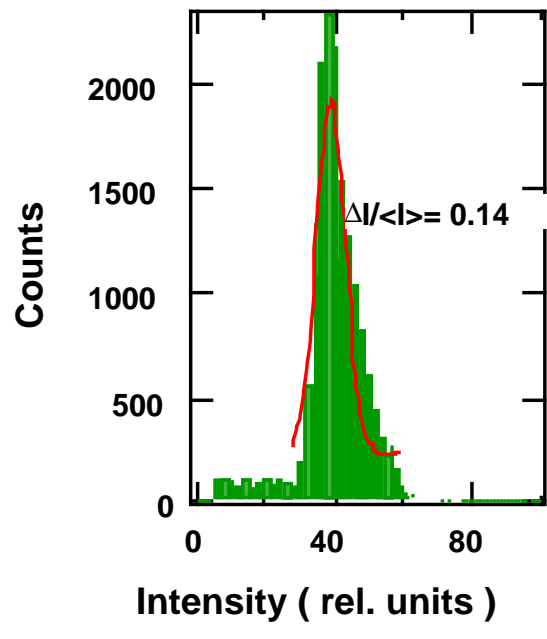

b.

Figure 9. Near field image at output of four-pass amplifier and histogram plot showing the distribution of intensities.

\section{CONCLUSIONS}

The current design of the preamplifier for the NIF laser system includes a diode-pumped regenerative amplifier and a flashlamp-pumped, four-pass power amplifier. The combined system can produce $14 \mathrm{~J}$ from a $200 \mathrm{pJ}$ input or a total gain of $7 \mathrm{X} 10^{10}$. This surpasses the original energetic specifications for the NIF laser system based upon the operation of 192 preamplifiers. Recent modifications to the specified number of preamplifiers may increase the required energy beyond the values demonstrated and described in this paper.

\section{REFERENCES}

1. "National Ignition Facility Design Report," Section1.1, UCRL-Prop-117093, L-17588-1, Lawrence Livermore National Laboratory, Livermore, CA, 1994.

2. R. Beach et. al., "Scalable diode-end-pumping technology applied to a 100-mJ Q-switched $\mathrm{Nd}^{3+}$ :YLF laser oscillator," Optics Letters 18, 1326 ( 1993).

3. J. T. Verdeyen, Laser Electronics 3rd edition, p.42, Prentice Hall, Englewood Cliffs, NJ, 1995.

4. L. M. Frantz and J. S. Nodvik, "Theory of pulse propagation in a laser amplifier," J. Appl. Phys. 84, $2346(1963)$.

5. A. E. Siegman, Lasers, University Science Books, Mill Valley, CA 1986.

Work performed under the auspices of the U. S. Department of Energy by Lawrence Livermore National Laboratory under contract no. W-7405-Eng-48. 


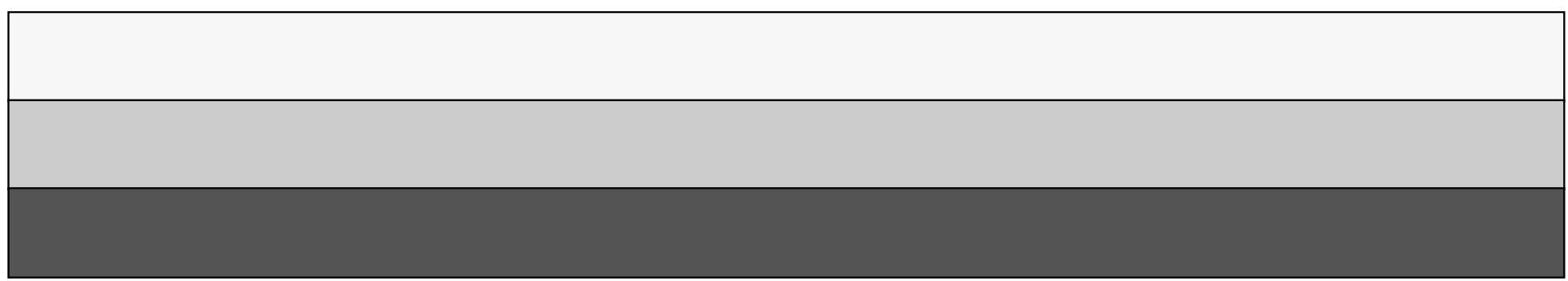

